\title{
A Simulation-Based Program for Preparedness for COVID-19 at a Pediatric Tertiary Hospital in Saudi Arabia
}

\author{
Tarek R. Hazwani ${ }^{1,2,3}$, Zahra Al Hassan ${ }^{4}$, Ahlam Al Zahrani ${ }^{4}$, Ayham Al Badawi ${ }^{5}$ \\ 1. Medical Research, King Abdullah International Medical Research Center, Riyadh, SAU 2. Pediatric Critical Care, King \\ Abdulaziz Medical City, Ministry of National Guard - Health Affairs, Riyadh, SAU 3. College of Medicine, King Saud Bin \\ Abdulaziz University for Health Sciences, Riyadh, SAU 4. Nursing, Ministry of National Guard - Health Affairs, Riyadh, \\ SAU 5. Infection Prevention and Control, Ministry of National Guard - Health Affairs, Riyadh, SAU
}

Corresponding author: Tarek R. Hazwani, tarekhazwani@gmail.com

\section{Abstract \\ Background}

COVID-19 has created major challenges for academic education and clinical training, as well as on routine, emergency, and elective patients who have been impacted by the health service's response to COVID-19. Simulation helps recognize and correct both active and latent threats in health institutions.

\section{Objectives}

This study aimed to describe the implementation steps, challenges, and solutions for simulations to make a difference in hospital operational readiness in the response to COVID-19.

\section{Methods}

We conducted a series of in situ simulations in different areas of the hospital to deal with COVID-19 patients. We designed seven scenarios to include different clinical situations of pediatric COVID-19 cases, such as emergency room triage and respiratory support.

\section{Results}

In total, seven simulation-based drills were conducted during March 1-30, 2020, and 89 healthcare professionals participated in these sessions. Many of the revealed latent threats were regarding teamwork, workflows, and adherence to infection control measures.

\section{Conclusion}

We found that in situ simulations helped to identify multiple latent hazard issues. Simulations have a great positive impact on hospital preparedness for the COVID-19 crisis in the pediatric field. Video-recorded simulations method is a good alternative to maintain medical supplies during the COVID-19 crisis.

Categories: Medical Simulation, Pediatrics

Keywords: simulation, covid-19, preparedness, health care, crisis

\section{Introduction}

The COVID-19 pandemic has created many challenges that have necessitated changes in working practices among the entire world population, and particularly in healthcare settings.

This pandemic has had a detrimental effect on routine, emergency, and elective patients who have been impacted by the health service's response to COVID-19. Further, patient and staff safety is essential in any health institution during this crisis, and the dynamic and quick progression of the COVID-19 pandemic requires a highly adaptable and resilient healthcare system to manage the rapid impact ahead before a lot of COVID-19 patients seek treatment [1].

Simulation helps recognize and correct both active and latent threats in the local environment and health institutions [2]. In situ simulations can identify multiple potential operational gaps, which allow decisionmakers to take necessary action in time [1]. There are now significant pieces of evidence demonstrating that simulation education interventions can produce changes in provider knowledge, attitudes, and skills in both the simulation laboratory and clinical settings [3-8]. Not only does simulation training provide learning at the individual level, but it also has an integral part to play in systems testing. Every session holds the potential to learn and improve on the systems level, and simulation can be a useful tool in the development of new standard operating procedures and policies needed to respond to a crisis [9]. 
During previous epidemics, such as Ebola, simulation programs had a major role in health system readiness and contributed effectively to the establishment of critical strategic and preparedness plans. These plans included the rapid development and utilization of a comprehensive simulation-based training program for healthcare professionals (HCPs) [10].

Second to identifying the need for training, simulation is a powerful tool in managing the COVID-19 crisis, as it can not only ensure patient safety but also provide a safe learning and training environment for HCPs to develop practical skills to deal with COVID-19. Simulation and online learning played a major role in dealing with COVID-19 in its early stages in China [11].

The main objective of our program was to assess the hospital operational readiness by conducting a series of in situ simulations in different areas of our hospital that deal with pediatric COVID-19 patients. In this report, we describe implementation steps, challenges, and solutions for simulation programs for HCPs to make a difference in the response to COVID-19 at a pediatric tertiary hospital in Saudi Arabia and share ways in which a simulation program can be utilized in healthcare organizations operating under pressure.

\section{Materials And Methods \\ Running simulations to improve preparedness for COVID-19}

King Abdullah Specialized Children's Hospital (KASCH) is the largest pediatric tertiary-care medical center under the Ministry of National Guard - Health Affairs (MNG-HA) in Saudi Arabia. Pediatric Simulation Training and Education Program (PediSTEP) in KASCH is a multidisciplinary simulation program that includes nursing services, different specialties of physicians, as well as other services, such as emergency services, respiratory therapy, and an infection control department. It offers different types of simulation programs such as high-fidelity and low-fidelity simulation, interprofessional simulation, procedural sessions, quality simulation-based programs, and in situ simulation.

Based on our program's endeavor to provide high-quality and efficient training and education for HCPs, we believe simulation can play a major role in readiness for the global COVID-19 crisis and any potential similar future pandemics.

COVID-19 has been spreading rapidly across the world and pediatric cases are increasing in our hospital, making immediate training and preparedness testing of utmost importance.

To assess hospital operational readiness, we conducted a series of in situ simulations in different areas of the hospital that dealt with pediatric COVID-19 patients, such as pediatric emergency room, operation room, pediatric intensive care unit, and pediatric wards. We involved in these sessions all HCPs who usually care and deal with pediatric patients, including physicians, nursing staff, respiratory therapists, anesthesia technicians, and radiology technicians.

Clinical, quality improvement, and infection control departments were engaged in this simulation program. The clinical departments that were directly involved with the effort included personnel from emergency medicine, pediatric critical care, operating room, and pediatric wards, as well as nursing services.

There were two major goals for this COVID-19 simulation program. First, simulation can play a role in understanding and testing the robustness of our systems such as infection control measures and flow of patients from emergency room to pediatric wards or pediatric intensive care unit, and optimizing workflows, dependencies, etc. (system focus). Second, simulation can help HCPs in safely and effectively handling patients with COVID-19, and in supporting HCPs in dealing with the emotional strain of the situation (personal focus).

Aims of our COVID-19 simulation program:

- To offer a simulation for HCPs to test and prepare them for a possible escalation of the COVID-19 situation.

- To prepare and reassure staff that the management of COVID-19 cases can be carried out safely.

- To reduce the cognitive load of HCPs as the workload escalates and to ensure a coordinated response.

To accomplish these goals, a COVID-19 simulation-based program was developed and run by PediSTEP team facilitators, including physicians, nurses, and infection control practitioners. We designed seven scenarios to include different clinical situations of pediatric COVID-19 cases, such as emergency room triage and respiratory support (Table 1 ). 


\section{Cureus}

\begin{tabular}{|c|c|c|}
\hline Scenario title & Environment & Target group \\
\hline $\begin{array}{l}\text { Aerostation/noninvasive } \\
\text { ventilation }\end{array}$ & Pediatric ward & Nurses, physicians, respiratory therapists \\
\hline Intubation & Pediatric intensive care unit, emergency room & $\begin{array}{l}\text { Physicians, nurses, respiratory therapists, radiology } \\
\text { technicians }\end{array}$ \\
\hline Intubation/extubation & Operation room & Physicians, nurses, anesthesia technicians \\
\hline Mechanical ventilation care & Pediatric intensive care unit & Physicians, nurses, respiratory therapists \\
\hline Iransportation & $\begin{array}{l}\text { From pediatric ward to pediatric intensive care } \\
\text { unit }\end{array}$ & Physiclans, nurses, respiratory therapists \\
\hline Cardiopulmonary resuscitation & Emergency room, pediatric intensive care unit & Physicians, nurses, respiratory therapists \\
\hline Triage & Emergency room & Nurses \\
\hline
\end{tabular}

\section{TABLE 1: COVID-19 simulation-based scenarios}

An evaluation template was formed by the PediSTEP team to unify the qualitative evaluation step. This evaluation form includes many items related to the team performance issue, infection control measures, and system issues. It rated the items as done, partially done, and not done, with a comments box for further notes. These simulations have been conducted by an expert team from PediSTEP members.

The simulations occurred during a normal working day and were run using a full-body manikin and the real settings and resources of the involved areas, which included the emergency department, pediatric wards, pediatric intensive care unit, and the operation room. These in situ simulations allowed us to include regular HCPs working that day within their real environment. The participating HCPs included physicians, nursing staff, respiratory therapists, anesthesia technicians, and radiology technicians.

After the simulation was completed, the participants were debriefed by PediSTEP facilitators.

\section{Results}

In total, seven simulation-based drills were conducted during March 1-30, 2020, and 89 HCPs participated in these sessions (Table 2).

\begin{tabular}{|l|l|}
\hline Participants & N (\%) \\
\hline Total & $89(100)$ \\
\hline Physicians & $17(19)$ \\
\hline Nursing staff & $56(63)$ \\
\hline Respiratory therapists & $10(11)$ \\
\hline Radiology technicians & $3(3)$ \\
\hline Anesthesia technicians & $3(3)$ \\
\hline
\end{tabular}

TABLE 2: Characteristics of healthcare providers who participated in COVID-19 simulation-based scenarios

These series of multidisciplinary in situ simulations exposed many issues that were qualitatively assessed and presented to related departments and administrators, so action plans were created. Many of the latent threats revealed by our simulations were regarding teamwork, workflows, and adherence to infection control measures, such as control of crowdedness inside the patient's room, proper donning and doffing of the personal protective equipment (PPE), equipment availability (e.g., a high-efficiency particulate air filter), and communication among the HCPs. 
These sessions received encouraging feedback through the PediSTEP electronic feedback form, which was distributed after each session. Most trainees revealed in their comments that the simulation exercises enhanced confidence and reduced anxiety among the pediatric HCPs during the COVID-19 crisis. Even while witnessing the impact of the virus on adult patients, the trainees reported that the exercises helped to normalize the processes required to care for pediatric patients with COVID-19 and afforded them an opportunity to become familiar with the organization's new policies.

\section{Discussion}

\section{Challenges and solutions}

We were planning to continue to use in situ simulation as the COVID-19 outbreak progresses, but this simulation is resource-consuming, in time, human resources, and medical supplies, especially during the current acute phase of a disease outbreak.

Our major challenge was the large number of HCPs that require training and the limited training resources of PPE, for which demand exceeds availability in most current healthcare systems, and these resources should be preserved for the real situations of the COVID-19 crisis, not consuming them in simulation training [12].

As these simulations were performed on the clinical unit during work hours, another issue raised is the need to balance training needs with the current work status of the participants. Therefore, to match needs, challenges, and methods to implement effective interventions, a novel way of enacting simulations was established. Thus, instead of in situ simulation sessions, we initiated video-recorded modules for each of these scenarios, which became available online through the PediSTEP webpage in the MNG-HA home page as E-learning modules for all KASCH staff (approximately 1,500 HCPs).

A spectrum of educational simulation-based online resources included different COVID-19 scenarios that were used to educate and verify both institutional and provider readiness in the identification and care of patients suspected of or diagnosed with COVID-19. This allowed us to ensure the appropriate use of resources, maintain homogeneous education, and enable effective patient care. This gave healthcare workers the opportunity to watch it in their free time or through their working hours and allow for self-paced learning.

There were several outcomes of this simulation program. First, it allowed us to identify potential pitfalls and challenges in the management of COVID-19 patients during their stay at our hospital. Second, the simulation program helped to increase the awareness and exploration of existing hospital guidelines and in developing new guidelines related to COVID-19. Third, it helped to improve the readiness of HCPs in crisis situations and in preparing them to protect themselves and save valuable resources.

The final program currently uses blended learning solutions to optimize efficiency and effectiveness. The initial vision was carried through the final design and consisted of requisite parts that included online webbased content and in situ training of simulation activities. We found that in situ simulations identified multiple latent hazard issues, and it allowed us to take corrective action before the COVID-19 crisis developed.

\section{Conclusions}

Simulation has great potential to positively impact hospital preparedness, training and educating HCPs in the pediatric field, and mitigating the negative effects of the COVID-19 crisis and for any future crisis situations. The video-recorded simulations method is a good alternative to in situ simulations to maintain medical supplies during the COVID-19 crisis.

We believe that this report may serve as an example for other simulation programs that are truly integrated with healthcare delivery systems and that can engage in significant, collaborative training development activities in response to operational needs.

\section{Additional Information \\ Disclosures}

Human subjects: All authors have confirmed that this study did not involve human participants or tissue. Animal subjects: All authors have confirmed that this study did not involve animal subjects or tissue. Conflicts of interest: In compliance with the ICMJE uniform disclosure form, all authors declare the following: Payment/services info: All authors have declared that no financial support was received from any organization for the submitted work. Financial relationships: All authors have declared that they have no financial relationships at present or within the previous three years with any organizations that might have an interest in the submitted work. Other relationships: All authors have declared that there are no other relationships or activities that could appear to have influenced the submitted work. 


\section{Acknowledgements}

We would like to acknowledge the help of the quality department, the Infection Prevention and Control Program, and the nursing service that aided in the running of the simulations. In particular, we would like to thank Dr. Yasser Kazzaz, Ms. Angela Caswell, Ms. Iris Murabi, Mr. Yahay Bakheet, and Mr. Yassine Kassem. We would like to thank Editage (www.editage.com) for English language editing.

\section{References}

1. Fregene TE, Nadarajah P, Buckley JF, Bigham S, Nangalia V: Use of in situ simulation to evaluate the operational readiness of a high-consequence infectious disease intensive care unit. Anaesthesia. 2020, 75:733-738. 10.1111/anae.15048

2. Brazil V: Translational simulation: not 'where?' but 'why?' A functional view of in situ simulation . Adv Simul (Lond). 2017, 2:20. 10.1186/s41077-017-0052-3

3. Brydges R, Hatala R, Zendejas B, Erwin PJ, Cook DA: Linking simulation-based educational assessments and patient-related outcomes: a systematic review and meta-analysis. Acad Med. 2015, 90:246-256.

10.1097/acm.0000000000000549

4. Shin S, Park JH, Kim JH: Effectiveness of patient simulation in nursing education: meta-analysis . Nurse Educ Today. 2015, 35:176-182. 10.1016/j.nedt.2014.09.009

5. Cook DA, Brydges R, Zendejas B, Hamstra SJ, Hatala R: Technology-enhanced simulation to assess health professionals: a systematic review of validity evidence, research methods, and reporting quality. Acad Med. 2013, 88:872-883. 10.1097/acm.0b013e31828ffdcf

6. Zendejas B, Brydges R, Wang AT, Cook DA: Patient outcomes in simulation-based medical education: a systematic review. J Gen Intern Med. 2013, 28:1078-1089. 10.1007/s11606-012-2264-5

7. Hazwani TR, Alosaimi A, Almutairi M, Shaheen N, Al Hassan Z, Antar M: The impact of mock code simulation on the resuscitation practice and patient outcome for children with cardiopulmonary arrest. Cureus. 2020, 12:e9197. 10.7759/cureus.9197

8. Hazwani TR, Harder N, Shaheen NA, et al.: Effect of a pediatric mock code simulation program on resuscitation skills and team performance. Clin Simul Nurs. 2020, 44:42-49. 10.1016/j.ecns.2020.01.004

9. Patterson MD, Geis GL, Falcone RA, LeMaster T, Wears RL: In situ simulation: detection of safety threats and teamwork training in a high risk emergency department. BMJ Qual Saf. 2013, 22:468-477. 10.1136/bmjqs-2012-000942

10. Phrampus PE, O’Donnell JM, Farkas D, Abernethy D, Brownlee K, Dongilli T, Martin S: Rapid development and deployment of Ebola readiness training across an academic health system: the critical role of simulation education, consulting, and systems Integration. Simul Healthc. 2016, 11:82-88. 10.1097/sih.0000000000000137

11. Li L, Lin M, Wang X, Bao P, Li Y: Preparing and responding to 2019 novel coronavirus with simulation and technology-enhanced learning for healthcare professionals: challenges and opportunities in China. BMJ Simul Technol Enhanc Learn. 2020, 6:196-198. 10.1136/bmjstel-2020-000609

12. Grasselli G, Pesenti A, Cecconi M: Critical care utilization for the covid-19 outbreak in Lombardy, Italy: early experience and forecast during an emergency response. JAMA. 2020, 323:1545-1546. 10.1001/jama.2020.4031 